\title{
Multidisciplinary therapy for scirrhous gastric cancer: a retrospective analysis and proposal of new treatment strategy
}

This article was published in the following Dove Press journal: Cancer Management and Research

\author{
Sachio Fushida' \\ Jun Kinoshita' \\ Katsunobu Oyama' \\ Takashi Fujimura ${ }^{2}$ \\ Tomoya Tsukada ${ }^{3}$ \\ Takahisa Yamaguchi' \\ Itasu Ninomiya' \\ Tetsuo Ohta' \\ 'Department of Gastroenterological \\ Surgery, Kanazawa University \\ Hospital, Kanazawa, Japan; \\ ${ }^{2}$ Department of Surgery, Toyama City \\ Hospital, Toyama, Japan; ${ }^{3}$ Department \\ of Surgery, Toyama Prefectural Central \\ Hospital, Toyama, Japan
}

Background: Scirrhous gastric cancer (SGC) is highly invasive and metastatic because of its interactions with stromal cells, such as fibroblasts and macrophages, and extracellular matrix, leading to a higher incidence of peritoneal metastasis (PM) than other gastric cancers (GCs). Taxane-based intraperitoneal chemotherapy (IPC) is a promising therapy for PM. We retrospectively analyzed outcomes of multidisciplinary therapies that included IPC for SGC.

Patients and therapy: Of 1,679 GC patients treated between 1990 and 2012, we analyzed 119 patients who underwent multidisciplinary therapy for SGC. Patients without PM received gastrectomy with lymphadenectomy and resection of involved adjacent organs followed by intraoperative IPC using cisplatin. Patients with PM received chemotherapy using fluorouracil, with or without methotrexate plus IPC using cisplatin before 2000, and S-1 plus IPC using paclitaxel or docetaxel since 2000.

Results: Of the 119 patients, 73 (61\%) had PM and 63 (53\%) had positive peritoneal lavage cytology. Of the 89 gastrectomy patients, 30 (34\%) had macroscopic residual tumors (R2). Of the patients treated since 2000, 66 (100\%) received S-1 plus taxanes and 44 patients (67\%) received taxane-based IPC. Median survival time was significantly longer in the post-2000 group (22.8 months) than in the pre-2000 group (9.5 months). In univariate analysis, lavage cytology, PM, taxane-based IPC, gastrectomy, and R2 resection were significant prognostic factors. However, only $\mathrm{R} 2$ resection was an independent prognostic factor in multivariate analysis (hazard ratio: 5.53, 95\% CI: 2.05-14.93).

Conclusion: As use of taxane-based IPC is not an independent prognostic factor, new multidisciplinary therapies are necessary to avoid $\mathrm{R} 2$ resections.

Keywords: peritoneal metastasis, intraperitoneal chemotherapy, taxanes

\section{Introduction}

Gastric cancer (GC) is the third most common cause of cancer deaths worldwide and is more common in East Asian countries. ${ }^{1}$ Scirrhous gastric cancer (SGC), also known as Borrmann type 4 or Linitis plastica, is a special type of gastric cancer, which is difficult to detect in its early stage because of its diffuse submucosal growth.,3 Despite recent advances in surgery and chemotherapy (CTX), outcomes of treatment for SGC have remained very poor., ${ }^{4,5}$ Although studies of neoadjuvant CTX against SGC have shown some positive effects, their survival benefits are less clear, because SGC patients often develop peritoneal metastasis (PM). ${ }^{6-8} \mathrm{PM}$ is relatively resistant to systemic CTX because of the poor blood supply and hypoxia of cancer cells in the peritoneum. ${ }^{9}$ Therefore, intraperitoneal chemotherapy (IPC) has been theoretically
Correspondence: Sachio Fushida

Department of Gastroenterological Surgery, Kanazawa University Hospital, 13-I Takara-machi, Kanazawa 920-864I, Japan

Email fushida@staff.kanazawa-u.ac.jp 
acceptable as regional intensive CTX to treat PM. Several studies have reported the effectiveness of taxane-based IPC for PM of GC, but its effectiveness in SGC has not been fully examined. ${ }^{10-12}$ In the current study, we retrospectively analyzed the outcomes of patients with SGC who were treated at our institution with multidisciplinary therapies. Also, we propose a new treatment strategy that considers the biological property of SGC, which is typified by interaction with stroma such as fibroblasts, macrophages, and extracellular matrix (ECM), resulting in organ fibrosis and chemoresistance.

\section{Patients and methods}

\section{Patients}

Of 1,679 patients treated for GC between January 1990 and December 2012 at the Department of Gastroenterological Surgery, Kanazawa University Hospital, 119 underwent multidisciplinary therapy for SGC. Patient data were collected retrospectively from medical records and patients who had distant metastasis other than PM were excluded.

The present study was in accordance with the ethical standards of the responsible committees on human experimentation (institutional and national) and with the Helsinki Declaration of 1964 and later versions. This study was approved by the Institutional Review Board of Kanazawa University Graduate School of Medical Sciences (Permission number 2833-1). Written informed consent was obtained from all patients.

\section{Diagnosis}

All patients were pathologically diagnosed with gastric adenocarcinoma. SGC was diagnosed from imaging and endoscopic analyses. Thoraco-abdomino-pelvic enhanced computed tomography scans was used to assess metastasis. Patients treated after April 2000 received diagnostic laparoscopies to detect PM and peritoneal cytology. Clinical staging was based on the Japanese Classification of Gastric Carcinoma. ${ }^{13}$

\section{CTX}

Prior to 2000, patients with apparently resectable SGC routinely underwent upfront gastrectomy, followed by intraoperative IPC using $150 \mathrm{mg}$ of cisplatin dissolved in $1 \mathrm{~L}$ of physiological saline for 60 minutes. They might also receive postoperative CTX using tegafur plus uracil, with or without mitomycin C (MMC), according to patient's status, whereas patients diagnosed with PM received systemic CTX using fluorouracil, with or without methotrexate, and IPC using cisplatin. Since 2000, patients diagnosed with PM (by staging laparoscopies) received systemic CTX using S-1, and IPC using taxanes (paclitaxel [PTX] or docetaxel). Postoperative CTX using S-1 plus taxane-based IPC was continued as long as possible.

\section{Surgery}

Among patients who did not receive induction CTX, surgery was performed 2-4 weeks after SGC was determined to be resectable. After induction CTX for PM, patients whose lavage cytology turned to negative underwent second-look laparoscopy. Among patients who judged resectable, surgery was performed 4-6 weeks after their last day of CTX. The surgical procedure was total gastrectomy, or distal gastrectomy for "antral-type" primary tumors, located in the lower stomach. Involved adjacent organs or localized peritoneal metastatic nodules were also resected for curative intent. Although the standard lymphadenectomy was an en bloc D2 resection, D1 dissection was also carried out for patients with $\mathrm{R} 2$ resections for $\mathrm{PM}$.

\section{Statistical analysis}

Patients were grouped by stage, initial treatment, surgical curability, and existence of PM. Survival was analyzed with the Kaplan-Meier method and compared using the log-rank test. Univariate and multivariate analyses were carried out using a Cox proportional regression hazard model. $P<0.05$ was considered significant.

\section{Results}

\section{Clinicopathological findings and treatment}

The characteristics of the 119 patients with SGC are summarized in Table 1. Their median age was 59 years (range: 14-87 years). Of the 119 patients, 73 (61.3\%) had PM and $63(52.9 \%)$ had positive cytology of peritoneal lavage fluid. None of the patients had stage Ia disease; 86 had stage IV disease. Of the 89 surgical patients, 30 patients had macroscopic residual tumors (R2). R2 resection rate

Table I Clinicopathological characteristics of patients

\begin{tabular}{ll}
\hline Median age, years (range) & $\mathbf{5 9}$ (I4-87) \\
\hline Sex (male/female) & $52 / 67$ \\
$\mathrm{PM}(\mathrm{PO} / \mathrm{PI})$ & $46 / 73$ \\
Lavage cytology (CYO/CYI) & $56 / 63$ \\
Clinical stage (la/lb/II/Illa/IIlb/IV) & $0 / 3 / 15 / 9 / 6 / 86$ \\
Gastrectomy (yes/no) & $89(\mathrm{RO}-\mathrm{I}: 59, \mathrm{R} 2: 30) / 30$ \\
Treatment period (-I999/2000-) & $53 / 66$ \\
\hline
\end{tabular}

Abbreviation: PM, peritoneal metastasis. 
before 2000 was $48 \%$ and after 2000 was $20 \%$. Since 2000 , 66 patients received newer anticancer drugs, such as S-1 and taxanes. Of these 66 patients, 44 underwent IPC using docetaxel or PTX.

\section{Survival and prognostic factors}

Median survival time (MST) was significantly longer in patients treated since 2000 (22.8 months) than prior to 2000 (9.5 months; $P=0.0013$; Figure 1 ). Prognosis was notably worse among patients with PM prior to 2000, whose overall survival (OS) rates were as follows: 1-year: 15\%, 3-year: 3\%, and 5-year: $0 \%$. The 46 patients without PM did not significantly differ in survival before and after 2000, but the half of them suffered peritoneal recurrences (Figure 2).

Survival curves by curability showed that the 5-year survival rate for patients who underwent R0-1 resections was $31 \%$, with an MST of 29.2 months, whereas survival in

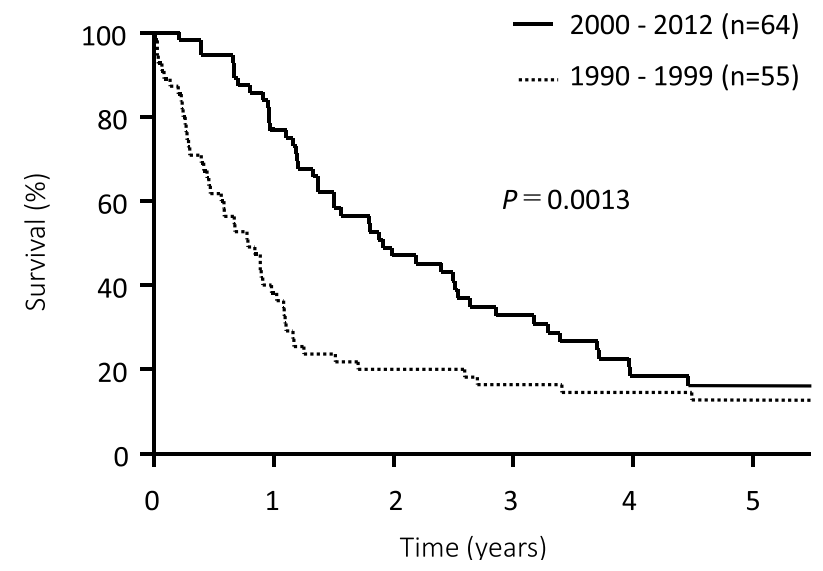

Figure I Kaplan-Meyer curve showing OS in all study patients. Abbreviation: OS, overall survival. patients who underwent $\mathrm{R} 2$ resections did not significantly differ from those who did not have gastrectomies (Figure 3).

OS rates by various prognostic factors among the 119 patients with SGC are shown in Table 2. In univariate analysis, PM, lavage cytology, CTX generation, gastrectomy, and $\mathrm{R} 2$ resection were shown as significant prognostic factors. In multivariate analysis, lavage cytology, generation of CTX, and $\mathrm{R} 2$ resection were shown as independent prognostic factors.

We also investigated various prognostic factors in the 66 patients who were treated since 2000 (Table 3). In univariate analysis, lavage cytology, PM, taxane-based IPC, gastrectomy, and R2 resection were significant prognostic factors; but in multivariate analysis only $\mathrm{R} 2$ resection was found to be an independent prognostic factor (hazard ratio: 5.530, 95\% CI: 2.049-14.925, $P=0.0007$ ).

\section{Discussion}

Because SGC is characterized by diffuse submucosal spreading and deep infiltration into the gastric wall, accompanied with fibrosis, serosal invasion is present in many cases at diagnosis. ${ }^{2}$ Therefore, PM is also often found at diagnosis, and prognosis is extremely poor. ${ }^{14}$ In this study, 73 patients $(61.3 \%)$ had PM; their median OS (11.3 months) was significant worse than for those without PM (28.4 months; $P<0.001)$.

As IPC was considered to have an enhanced anticancer effect through high regional drug concentration, cisplatin was administered intraperitoneally for patients with PM before 2000. However, the MST of these patients before 2000 (5.6 months) was significantly worse than since 2000 (14.6 months; $P<0.001)$. Treatment between these two time

$-2000-2012$

…. 1990 - 1999

P $1(n=73)$

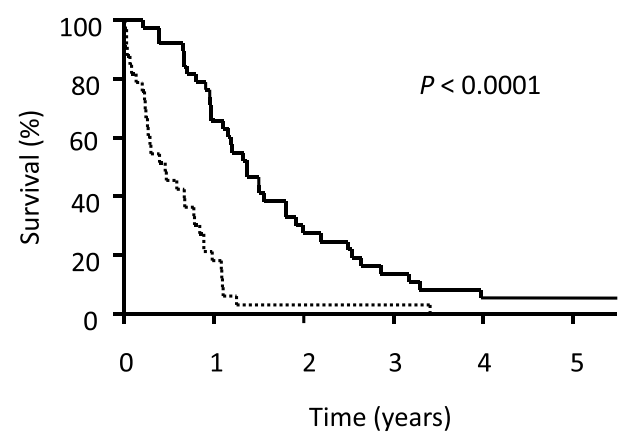

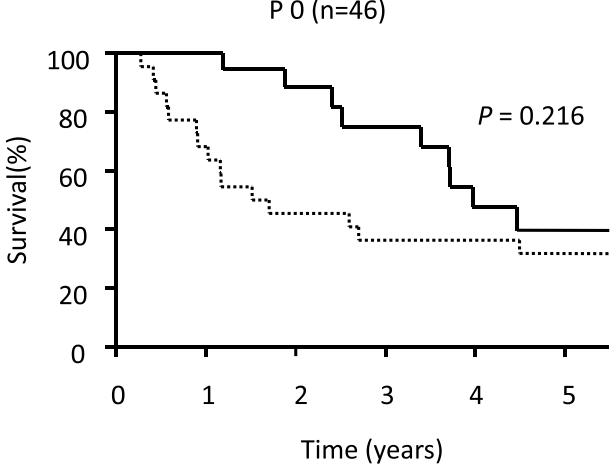

Figure 2 Kaplan-Meyer curve showing OS rates in patients with or without PM.

Abbreviations: $\mathrm{PI}$, peritoneal metastasis positive; $\mathrm{PO}$, peritoneal metastasis negative; OS, overall survival. 


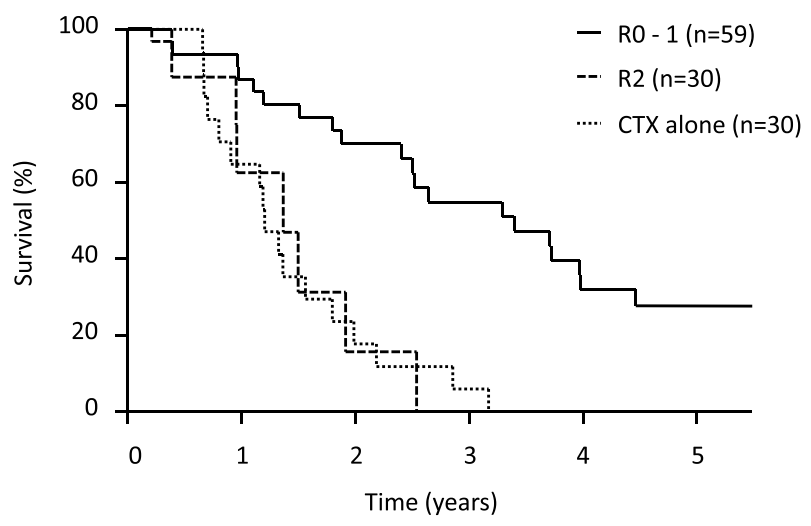

Figure 3 Kaplan-Meyer curve showing OS in patients with gastrectomy and those with CTX alone.

Abbreviations: R0-I, gastrectomy without macroscopic residual tumor; R2, gastrectomy with macroscopic residual tumor; CTX, chemotherapy; OS, overall survival. frames differed by the drugs used for IPC. Ideal agents for intraperitoneal administration have low peritoneal clearance and high area under the curve ratios from the peritoneal cavity to the plasma. Because cisplatin is hydrophilic and has a low molecular weight, it transports readily into the systemic circulation..$^{15}$ Although MMC is widely used in heated IPC, MMC also transports into systemic circulation. Moreover, IPC using MMC can induce encapsulated peritoneal fibrosis because of its chemical toxicity. ${ }^{9}$ In contrast, as taxanes are hydrophobic and have high molecular weights, they are retained much longer in the peritoneal cavity. ${ }^{16,17}$ IPC cannot achieve reduction of PM in only a few times, whereas taxanebased IPC can be administered repeatedly without peritoneal adhesion because of its antifibrotic effect. Intraperitoneal

Table 2 Cox hazard model analysis for OS (all patients)

\begin{tabular}{|c|c|c|c|c|c|c|}
\hline & \multicolumn{3}{|c|}{ Univariate analysis } & \multicolumn{3}{|c|}{ Multivariate analysis } \\
\hline & HR & $95 \% \mathrm{Cl}$ & $P$-value & HR & $95 \% \mathrm{Cl}$ & $P$-value \\
\hline \multicolumn{7}{|l|}{ Sex } \\
\hline Male/female & 0.793 & $0.533-1.180$ & 0.252 & & & \\
\hline \multicolumn{7}{|l|}{$\mathrm{CY}(+)^{\mathrm{a}}$} \\
\hline Yes/no & 2.712 & $1.793-4.100$ & $<0.0001$ & 1.686 & I.020-2.786 & $0.04 I$ \\
\hline \multicolumn{7}{|l|}{$\mathrm{P}(+)^{\mathrm{b}}$} \\
\hline Yes/no & 3.561 & $2.254-5.624$ & $<0.0001$ & 1.689 & $0.839-3.401$ & 0.142 \\
\hline \multicolumn{7}{|l|}{ CTX } \\
\hline New/oldc & 0.524 & $0.35 \mathrm{I}-0.782$ & 0.0016 & 0.372 & $0.235-0.589$ & $<0.0001$ \\
\hline \multicolumn{7}{|l|}{$\mathrm{IPC}^{\mathrm{d}}$} \\
\hline Yes/no & 0.764 & $0.487-1.199$ & 0.243 & & & \\
\hline \multicolumn{7}{|l|}{ Taxane IPe } \\
\hline Yes/no & 0.985 & $0.655-|.48|$ & 0.941 & & & \\
\hline \multicolumn{7}{|l|}{ Gastrectomy } \\
\hline Yes/no & 0.399 & $0.254-0.627$ & $<0.0001$ & I.157 & $0.655-2.045$ & 0.615 \\
\hline \multicolumn{7}{|l|}{$\mathrm{R} 2$ resection } \\
\hline Yes/no & 5.686 & $3.500-9.237$ & $<0.0001$ & 4.332 & $2.092-8.970$ & $<0.0001$ \\
\hline
\end{tabular}

Notes: aPeritoneal cytology positive. 'PPM positive. 'New: 2000-2013, old: 1990-1999. dPC. eIPC using PTX or docetaxel.

Abbreviations: OS, overall survival; HR, hazard ratio; CTX, chemotherapy; IPC, intraperitoneal chemotherapy; PM, peritoneal metastasis; PTX, paclitaxel.

Table 3 Cox hazard model analysis for OS among patients treated since $2000(n=66)$

\begin{tabular}{|c|c|c|c|c|c|c|}
\hline & \multicolumn{3}{|c|}{ Univariate analysis } & \multicolumn{3}{|c|}{ Multivariate analysis } \\
\hline & HR & $95 \% \mathrm{Cl}$ & $P$-value & HR & $95 \% \mathrm{Cl}$ & $P$-value \\
\hline \multicolumn{7}{|l|}{ Sex } \\
\hline Male/female & 0.819 & $0.459-1.458$ & 0.499 & & & \\
\hline \multicolumn{7}{|l|}{$\mathrm{CY}(+)^{\mathrm{a}}$} \\
\hline Yes/no & 2.483 & $1.380-4.467$ & 0.0024 & 1.635 & $0.778-3.436$ & 0.194 \\
\hline \multicolumn{7}{|l|}{$P(+)^{b}$} \\
\hline Yes/no & 3.456 & I.798-6.644 & 0.0002 & 1.10 & $0.437-2.816$ & 0.827 \\
\hline \multicolumn{7}{|l|}{ Taxane IPc } \\
\hline Yes/no & 3.109 & $1.490-6.484$ & 0.0025 & 1.725 & $0.723-4.119$ & 0.219 \\
\hline \multicolumn{7}{|l|}{ Gastrectomy } \\
\hline Yes/no & 0.268 & $0.142-0.507$ & $<0.0001$ & 1.448 & $0.597-3.510$ & 0.413 \\
\hline \multicolumn{7}{|l|}{$\mathrm{R} 2$ resection } \\
\hline Yes/no & 5.671 & $2.846-11.299$ & $<0.0001$ & 5.530 & $2.049-14.925$ & 0.0007 \\
\hline
\end{tabular}

Notes: aPeritoneal cytology positive. 'PPM positive. 'IPC using PTX or docetaxel.

Abbreviations: OS, overall survival; HR, hazard ratio; PM, peritoneal metastasis; IPC, intraperitoneal chemotherapy; PTX, paclitaxel. 
administration of PTX or docetaxel improved MST by more than 20 months and increased the 1-year survival rate to more than $70 \%$ among GC patients with PM. ${ }^{10-12}$ The oral anticancer drug S-1 is a fluoropyrimidine derivative that combines tegafur with two modulators. ${ }^{18} \mathrm{~S}-1$ is also highly effective against $\mathrm{PM}$ as it facilitates higher concentrations of 5-FU and CDHP in peritoneal tumors than in plasma. ${ }^{19}$ For the above reasons, treatment outcomes since 2000 for the patients with PM have been excellent.

Taxane-based IPC combined with systemic CTX is a promising treatment modality for PM in GC. Although use of cytoreductive surgery plus heated IPC for PM in GC led to a 1 -year OS rate of only about $50 \%$, taxane-based IPC combined with systemic CTX resulted in a 1-year OS rate above $70 \% .^{20-22}$

However, taxane-based IPC was not a prognostic factor for SGC in this study. For the patients with severe PM, tumors did not always disappear by taxane-based IPC. Such patients did not receive surgery, resulting in poor prognosis. On the other hand, taxane-based IPC was performed as adjuvant CTX after 2000; however, there was no significant difference in survival between before and after 2000 in patients without PM. Takahashi et $\mathrm{al}^{23}$ also failed to prove superiority of intraperitoneal PTX over intravenous PTX delivered after surgery in gastric linitis. These results support the idea that postoperative adhesion might inhibit delivery of intraperitoneal taxane. Furthermore, the biological properties of SGC make treatment challenging. PM from SGC is characterized by diffusely infiltrating and proliferating cancer cells, accompanied by extensive stromal fibrosis that impedes drug delivery and results in chemoresistance. ${ }^{24}$

Of the 119 patients, 89 (74.8\%) underwent surgical treatment and 30 patients received noncurative resection (R2). In this study, $\mathrm{R} 2$ resection was the independent prognostic factor most associated with poor outcome in multivariate analysis. After gastrectomy, residual tumor growth could be promoted by inducing inflammatory cytokines and immunosuppression. ${ }^{25}$ Moreover, most patients who received surgical treatment needed total gastrectomy because of diffuse expansion of SGC. In this study, only 4 of 89 patients underwent partial gastrectomy (data not shown). Compliance with CTX after total gastrectomy is generally lower than with any other type of gastrectomy because of postoperative weight loss. ${ }^{26,27}$ These results support the idea that OS after R2 resection did not differ from CTX alone. The REGATTA trial, which investigated the superiority of gastrectomy followed by CTX vs CTX alone in patients with noncurable GC, also showed gastrectomy could not be justified for treatment of patients with noncurable $\mathrm{GC}^{28}$
To avoid R2 resection, new treatment strategies are needed to target tumor proliferation and fibrosis in SGC. Recently, the effectiveness of anti- VEGFR2 antibody, with or without PTX, has been reported in patients with metastatic GC. ${ }^{29,30}$ Blocking VEGFR2 not only inhibits tumor growth directly but also normalizes tumor vessels and improves the delivery of small nanoparticles such as nab-PTX. ${ }^{31}$ Bando et $\mathrm{l}^{32}$ reported favorable efficacy for nab-PTX combined with ramucirumab in patients with previously treated advanced GC, including $43 \%$ of those with PM. ${ }^{32}$ Intratumoral fibrosis in SGC leads to poor drug delivery as a result of interstitial high pressure. Antifibrotic agents are therefore expected to improve drug delivery. TGF- $\beta$ has been shown to be a key mediator of tissue fibrosis. ${ }^{33,34}$ We previously demonstrated that GC has higher levels of both angiotensin II and AT1 receptor than normal tissues. ${ }^{35}$ This angiotensin II/AT1 receptor axis contributes to tissue fibrosis through TGF- $\beta$ production in the cancer microenvironment. Okazaki et $\mathrm{al}^{36}$ reported that candesartan, which is an AT1 blocker, inhibited tumor proliferation and stromal fibrosis derived from GC xenografts. Tranilast, which is used as an antiallergic and antifibrotic agent, is also expected to function as antifibrotic agent by suppressing the TGF- $\beta$ signaling pathway. ${ }^{37}$

Because tumors with $\mathrm{R} 0$ resections are regarded to be relatively chemosensitive, combination therapy based on its molecular mechanism between stroma and cancer cells should be developed to increase chemosensitivity. Larger multicenter prospective studies are required to clarify the efficacy of new multidisciplinary therapy, using small-molecule PTX plus anti-VEGFR antibodies, including antifibrotic agents.

There are several limitations in this study. First, treatment efficacy could only be evaluated by OS rate, because PM is regarded as an unmeasurable lesion. Second, our study was small, and conducted in a single institution; therefore, further larger, multicenter studies are required to validate our results.

\section{Conclusion}

As use of taxane-based IPC is not an independent prognostic factor, new multidisciplinary therapies are necessary to avoid $\mathrm{R} 2$ resections.

\section{Acknowledgments}

We are grateful to members of the Department of Gastroenterological Surgery of Kanazawa University Hospital for their helpful suggestions. We thank Marla Brunker, from Edanz Group for editing a draft of this manuscript.

\section{Disclosure}

The authors report no conflicts of interest in this work. 


\section{References}

1. Orditura M, Galizia G, Sforza V, et al. Treatment of gastric cancer. World J Gastroenterol. 2014;20(7):1635-1649.

2. Kanter MA, Isaacson NH, Knoll AM, Nochomovitz LE. The diagnostic challenge of metastatic linitis plastic. Two cases and a consideration of the problem. Am Surg. 1986;52(9):510-513.

3. Park MS, Ha HK, Choi BS, et al. Scirrhous gastric carcinoma: endoscopy versus upper gastrointestinal radiography. Radiology. 2004;231(2):421-426.

4. Furukawa H, Hiratsuka M, Iwanaga T, et al. Extended surgery - left upper abdominal exenteration plus Appleby's method - for type 4 gastric carcinoma. Ann Surg Oncol. 1997;4(3):209-214.

5. Suga S, Iwase H, Shimada M, et al. Neoadjuvant chemotherapy in scirrhous cancer of the stomach using uracil and tegafur and cisplatin. Intern Med. 1996;35(12):930-936.

6. Takahashi S, Kinoshita T, Konishi M, et al. Phase II study of sequential high-dose methotrexate and fluorouracil combined with doxorubicin as a neoadjuvant chemotherapy for scirrhous gastric cancer. Gastric Cancer. 2001;4(4):192-197.

7. Kinoshita T, Sasako M, Sano T, et al. Phase II trial of S-1 for neoadjuvant chemotherapy against scirrhous gastric cancer (JCOG 0002). Gastric Cancer. 2009;12(1):37-42.

8. Kunisaki C, Makino H, Kimura J, et al. Impact of S-1 plus cisplatin neoadjuvant chemotherapy on scirrhous gastric cancer. Oncology. 2015;88(5):281-288.

9. Fushida S, Oyama K, Kinoshita J, et al. Intraperitoneal chemotherapy as a multimodal treatment for gastric cancer patients with peritoneal metastasis. J Cancer Ther. 2013;04(09):6-15.

10. Ishigami H, Kitayama J, Kaisaki S, et al. Phase II study of weekly intravenous and intraperitoneal paclitaxel combined with S-1 for advanced gastric cancer with peritoneal metastasis. Ann Oncol. 2010;21(1):67-70.

11. Fujiwara Y, Takiguchi S, Nakajima K, et al. Intraperitoneal docetaxel combined with S-1 for advanced gastric cancer with peritoneal dissemination. J Surg Oncol. 2012;105(1):38-42.

12. Fushida S, Kinoshita J, Kaji M, et al. Phase I/II study of intraperitoneal docetaxel plus S-1 for the gastric cancer patients with peritoneal carcinomatosis. Cancer Chemother Pharmacol. 2013;71(5):1265-1272.

13. Japanese Gastric Cancer Association. Japanese Classification of Gastric Carcinoma - 2nd English Edition. Gastric Cancer. 1998;1(1):10-24.

14. Otsuji E, Kuriu Y, Okamoto K, et al. Outcome of surgical treatment for patients with scirrhous carcinoma of the stomach. Am J Surg. 2004;188(3):327-332.

15. Markman M. Intraperitoneal antineoplastic drug delivery: rationale and results. Lancet Oncol. 2003;4(5):277-283.

16. Ishigami H, Kitayama J, Otani K, et al. Phase I pharmacokinetic study of weekly intravenous and intraperitoneal paclitaxel combined with S-1 for advanced gastric cancer. Oncology. 2009;76(5):311-314.

17. Fushida S, Kinoshita J, Yagi M, et al. Dual anticancer effects of weekly intraperitoneal docetaxel in treatment of advanced gastric cancer patients with peritoneal carcinomatosis: a feasible and farmacokinetic study. Oncology Rep. 2008;19(5):1305-1310.

18. Shirasaka T, Nakano K, Takechi T, et al. Antitumor activity of $1 \mathrm{M}$ tegafur-0.4 M 5-chloro-2,4-dihydroxypyridine-1 M potassium oxonate (S-1) against human colon carcinoma orthotopically implanted into nude rats. Cancer Res. 1996;56(11):2602-2606.

19. Oshima T, Yamada R, Hatori S, Kunisaki C, Imada T. Pharmacokinetics of S-1 in patients with peritoneal dissemination of gastric cancer. Oncol Rep. 2006;16(2):361-366.

20. Glehen O, Gilly FN, Arvieux C, et al. Peritoneal carcinomatosis from gastric cancer: a multi-institutional study of 159 patients treated by cytoreductive surgery combined with perioperative intraperitoneal chemotherapy. Ann Surg Oncol. 2010;17(9):2370-2377.
21. Yang XJ, Huang CQ, Suo T, et al. Cytoreductive surgery and hyperthermic intraperitoneal chemotherapy improves survival of patients with peritoneal carcinomatosis from gastric cancer: final results of a phase III randomized clinical trial. Ann Surg Oncol. 2011;18(6):1575-1581.

22. Magge D, Zenati M, Mavanur A, et al. Aggressive locoregional surgical therapy for gastric peritoneal carcinomatosis. Ann Surg Oncol. 2014;21(5):1448-1455.

23. Takahashi N, Kanda M, Yoshikawa T, et al. A randomized phase II multicenter trial to explore efficacy of weekly intraperitoneal in comparison with intravenous paclitaxel administered immediately after gastrectomy to the patients with high risk of peritoneal recurrence: final results of the INPACT trial. Gastric Cancer. In press 2018.

24. Yashiro M, Chung YS, Nishimura S, Inoue T, Sowa M. Fibrosis in the peritoneum induced by scirrhous gastric cancer cells may act as "soil" for peritoneal dissemination. Cancer. 1996;77(8 Suppl):1668-1675.

25. Goldfarb Y, Sorski L, Benish M, Levi B, Melamed R, Ben-Eliyahu S. Improving postoperative immune status and resistance to cancer metastasis: a combined perioperative approach of immunostimulation and prevention of excessive surgical stress responses. Ann Surg. 2011;253(4):798-810.

26. Chou WC, Chang CL, Liu KH, et al. Total gastrectomy increases the incidence of grade III and IV toxicities in patients with gastric cancer receiving adjuvant TS-1 treatment. World J Surg Oncol. 2013;11:287.

27. Aoyama T, Sato T, Segami K, et al. Risk factors for the loss of lean body mass after gastrectomy for gastric cancer. Ann Surg Oncol. 2016;23(6):1963-1970.

28. Fujitani K, Yang HK, Mizusawa J, et al. Gastrectomy plus chemotherapy versus chemotherapy alone for advanced gastric cancer with a single non-curable factor (REGATTA): a phase 3, randomised controlled trial. Lancet Oncol. 2016;17(3):309-318.

29. Wilke H, Muro K, Van Cutsem E, et al. Ramucirumab plus paclitaxel versus placebo plus paclitaxel in patients with previously treated advanced gastric or gastro-oesophageal junction adenocarcinoma (RAINBOW): a double-blind, randomised phase 3 trial. Lancet Oncol. 2014;15(11):1224-1235.

30. Fuchs CS, Tomasek J, Yong CJ, et al. Ramucirumab monotherapy for previously treated advanced gastric or gastro-oesophageal junction adenocarcinoma (REGARD): an international, randomised, multicentre, placebo-controlled, phase 3 trial. Lancet. 2014;383(9911): 31-39.

31. Chauhan VP, Stylianopoulos T, Martin JD, et al. Normalization of tumour blood vessels improves the delivery of nanomedicines in a size-dependent manner. Nat Nanotechnol. 2012;7(6):383-388.

32. Bando H, Shimodaira H, Fujitani K, et al. A phase II study of nabpaclitaxel in combination with ramucirumab in patients with previously treated advanced gastric cancer. Eur J Cancer. 2018;91:86-91.

33. Xu J, Lamouille S, Derynck R. TGF-beta-induced epithelial to mesenchymal transition. Cell Res. 2009;19(2):156-172.

34. Fuyuhiro Y, Yashiro M, Noda S, et al. Upregulation of cancer-associated myofibroblasts by TGF- $\beta$ from scirrhous gastric carcinoma cells. $\mathrm{Br} J$ Cancer. 2011;105(7):996-1001.

35. Kinoshita J, Fushida S, Harada S, et al. Local angiotensin II-generation in human gastric cancer: correlation with tumor progression through the activation of ERK1/2, NF-kappaB and survivin. Int J Oncol. 2009;34(6):1573-1582.

36. Okazaki M, Fushida S, Harada S, et al. The angiotensin II type 1 receptor blocker candesartan suppresses proliferation and fibrosis in gastric cancer. Cancer Lett. 2014;355(1):46-53.

37. Saito H, Fushida S, Harada S, et al. Importance of human peritoneal mesothelial cells in the progression, fibrosis, and control of gastric cancer: inhibition of growth and fibrosis by tranilast. Gastric Cancer. 2018;21(1):55-67. 
Cancer Management and Research

\section{Publish your work in this journal}

Cancer Management and Research is an international, peer-reviewed open access journal focusing on cancer research and the optimal use of preventative and integrated treatment interventions to achieve improved outcomes, enhanced survival and quality of life for the cancer patient

The manuscript management system is completely online and includes

Submit your manuscript here: https://www.dovepress.com/cancer-management-and-research-journal 